\title{
ANÁLISIS COMPARATIVO ENTRE LOS ANÁLOGOS DE LA LHRH DE ADMINISTRACIÓN SEMESTRAL EN EL TRATAMIENTO DEL CÁNCER DE PRÓSTATA
}

\author{
Felipe Herranz Amo
}

Servicio de Urología. Hospital General Universitario Gregorio Marañón. Madrid. España.

Resumen.- OBJETIVO: Realizar un análisis comparativo de la eficacia y la seguridad de los análogos de la LHRH de administración semestral con indicación para el tratamiento del cáncer de próstata.

MÉTODO - Búsqueda en la base de datos PubMed de los ensayos clínicos (clinicals trials) publicados en el período 2006-2009 con las siguientes palabras claves: "prostate cancer" $y$ "triptorelin o leuprorelin" $y$ "6 month depot".

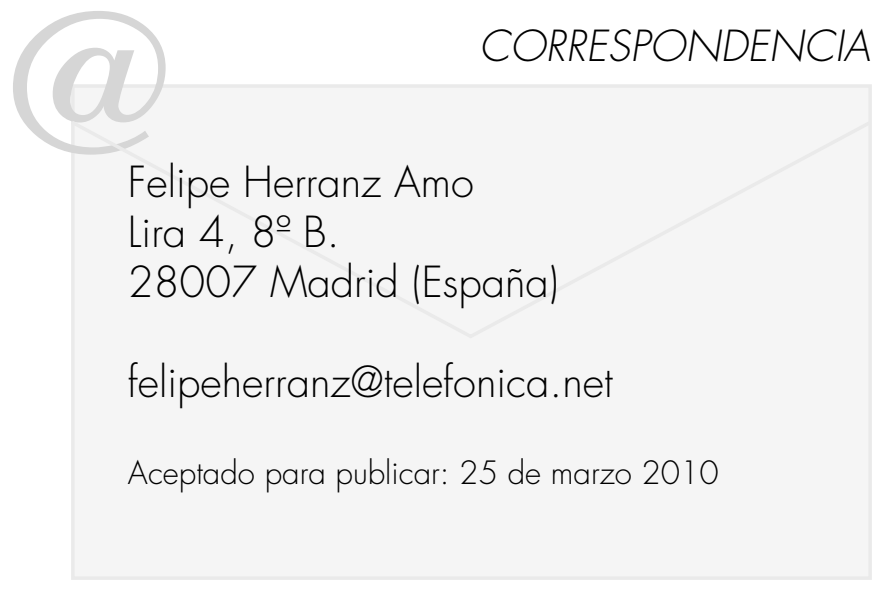

RESULTADOS - Los 3 análogos de la LHRH de administración semestral autorizados en la actualidad tienen una elevada eficacia (96-98\%) en la disminución de los niveles de testosterona por debajo de los $50 \mathrm{ng} / \mathrm{dl}$. Debido a la heterogeneidad de los pacientes incluidos en los 3 ensayos y a la variabilidad en la forma de presentar los resultados, no es posible comparar los "escapes" de testosterona ni su eficacia sobre los niveles de PSA. Se comunicaron una elevada incidencia de acontecimientos adversos (AA) en los 3 estudios, aunque solo fueron graves entre el 0,9\% y el 15,8\%. Solo en un estudio se comunicó la retirada de pacientes (2,5\%) por AA relacionados con el fármaco.

CONCLUSIÓN - A pesar de que en los 3 estudios existe una importante variabilidad del análisis y la forma de expresar los datos, no parece que existan grandes diferencias en cuanto a eficacia y seguridad.

Palabras clave: Cáncer de próstata. Análogo de la LHRH. Administración semestral. Eficacia. Seguridad.

Summary.- OBJECTIVES: To perform comparative analysis of the efficacy and the safety of six months formulation of LHRH analogues indicated for prostate cancer treatment.

METHOD: Search in the PubMed database for clinical trials published between 2006 and 2009 using the following key words: "prostate cancer", "triptorelin or leuprorelin" and "6-month depot".

RESULTS: The efficacy of all 3 six months formulation of LHRH analogues currently approved is high (96-98\%) for reducing testosterone levels down to below $50 \mathrm{ng} / \mathrm{dl}$. 
As the patients included in the three trials are quite heterogeneous, and due to the variability in the way of presenting results, it is not possible to compare testosterone escapes and their effect on PSA levels. The incidence of adverse events (AE) reported across the three trials was high, but only $0.9 \%$ to $15.8 \%$ were severe. Only one trial reported patient withdrawal (2.5\%) because of drug-related AEs.

CONCLUSION: Even though all the studies show and important variability in the analysis and data management, no significant efficacy and safety differences seem to exist.

Keywords: Prostate cancer. LHRH analogue. Semiannual administration. Efficacy. Safety.

\section{INTRODUCCIÓN}

Según la estimación que realiza anualmente la American Cancer Society (ACS) (1), el cáncer de próstata es el tumor más incidente en el varón y representa la $2^{\mathrm{a}}$ causa de muerte tumoral.

Desde la generalización de la determinación del PSA para el diagnóstico precoz del cáncer de próstata, hemos observado una migración del estadio tumoral al diagnóstico hacia las formas clínicas localizadas. Así en el estudio sobre la incidencia del cáncer de próstata en la Comunidad de Madrid del año 2000 (2), el $75 \%$ de los pacientes se diagnosticaron en un estadio localizado, el 12,5\% localmente avanzado y otro $12,5 \%$ con metástasis.

La supresión de los niveles de testosterona circulantes es el tratamiento paliativo más utilizado en los pacientes con enfermedad metastática. La guía de práctica clínica de la Asociación Europea de Urología (EAU) (3) recomienda el tratamiento hormonal en los pacientes con enfermedad metastática $M+y / o$ $\mathrm{N}+$ (grado de recomendación $\mathrm{A}$ ), en la enfermedad localmente avanzada (paciente sintomático, PSA > $25-50 \mathrm{ng} / \mathrm{ml}$ y PSA-DT < 1 año) o como adyuvancia a la radioterapia (grado de recomendación A) $y$ en la enfermedad localizada de alto riesgo como neoadyuvancia y concomitancia a la radioterapia (grado de recomendación A). La guía de práctica clínica del National Comprehensive Cancer Neetwork (NCCN) (4), añade a las anteriores recomendaciones la utilización en la enfermedad localizada de riesgo intermedio como neoadyuvancia y concomitancia a la radioterapia.

Los métodos más utilizados para la supresión de la testosterona son la orquiectomía bilateral y la utilización de análogos de la LHRH. En un estudio poblacional realizado sobre la base de datos de Medicare en el periodo 1993-2000 se observó un aumento de la prevalencia de la supresión de los niveles de testosterona en el tratamiento del cáncer de próstata (de $1,8 \%$ a $2,9 \%, p<0,001$ ), a expensas de la utilización de los análogos de la LHRH ya que la orquiectomía bilateral disminuyó del $53 \%$ en 1999 al $21 \%$ en el año 2000 (5). Probablemente la introducción de la hormonoterapia como adyuvancia y concomitancia a la radioterapia haya aumentado de forma considerable la prevalencia de la hormonoterapia en la actualidad.

En 1941 Huggins y Hodges (6) publicaron la relación existente entre la orquiectomía y el cáncer de próstata. Por el descubrimiento de la dependencia hormonal del cáncer de próstata se le otorgó a Huggins el premio Nobel en 1966. En 1971 Schally y Guillemin aislaron la estructura molecular de la LHRH y en 1973 sintetizaron el primer análogo del la LHRH, la triptorelina, mediante la sustitución de un aminoácido en la posición 6 de su estructura, recibiendo el premio Nobel en 1977. En 1980 Labrie, Coy y Schally utilizaron análogos de la LHRH en pacientes con cáncer de próstata y en 1989 la FDA aprobó la utilización mensual de los análogos en el cáncer de próstata. Desde entonces se han sintetizado varios análogos de la LHRH.

Los agonistas de la LHRH tienen una semivida de eliminación corta (7), por esta razón se comenzó administrándolos diariamente de forma subcutánea - intranasal. La incorporación del péptido a un polímero biocompatible y biodegradable ha permitido que se prolongue el tiempo de administración a 1, 2, $3,4,6$ y 12 meses (8), facilitando su aplicación por parte del facultativo y el cumplimiento del tratamiento por parte del paciente.

El objetivo de este estudio es realizar un análisis comparativo sobre la eficacia y la seguridad de los análogos de la LHRH que se administran semestral en el tratamiento del cáncer de próstata.

\section{MATERIAL Y MÉTODO}

Búsqueda en la base de datos PubMed de los ensayos clínicos (clinicals trials) publicados en el período 2006-2009 con las siguientes palabras claves: "prostate cancer" $y$ "triptorelin o leuprorelin" $y$ "6 month depot".

Hemos encontrado 3 ensayos clínicos que reunían las condiciones de búsqueda y que respondían al objetivo planteado en el estudio (9-1 1). 
Se realiza un análisis comparativo de la eficacia y la seguridad de estos estudios. En el análisis sobre la eficacia se analizan la reducción de los niveles de testosterona sérica y del PSA. En el análisis sobre la seguridad se evalúan los acontecimientos adversos.

\section{RESULTADOS}

\section{Tipos de estudio}

- El estudio con leuprorelina subcutánea (SC) (9) fue un ensayo clínico en fase III, abierto, multicéntrico con una duración de 12 meses ( 1 inyección cada 6 meses). Los objetivos del estudio fueron la seguridad y la eficacia.

- El estudio con triptorelina (1 1) fue un ensayo clínico en fase III, abierto, multicéntrico con una duración de 12 meses (1 inyección cada 6 meses). Los objetivos del estudio fueron la eficacia, la seguridad y la farmacocinética.

- El estudio con leuprolerina intramuscular (IM) (10) fue un ensayo clínico en fase III en el que se realizó una aleatorización a recibir una dosis de 11,25 mg del fármaco cada 3 meses, $22,5 \mathrm{mg}$ cada 6 meses o $30 \mathrm{mg}$ cada 6 meses durante 12 meses. Los objetivos principales de este estudio fueron la seguridad y la tolerabilidad, como objetivo secundario se analizó la eficacia entre las tres ramas de aleatorización del estudio.

\section{Tamaño muestral y criterios de inclusión (Tabla I)}

- En el estudio con leuprorelina SC se incluyeron 111 pacientes, con diagnostico histológico de cáncer, estadio mayor a T1, estado general OMS 0-2 y esperanza de vida superior a un año. Solo el $17 \%$ tenían un cáncer de próstata localmente avanzado (estadio C) y el $40 \%$ presentaban metástasis (estadio D) en el momento del diagnóstico. La media de PSA al inicio del estudio fue de $39,8 \mathrm{ng} / \mathrm{ml}(0,19-2.284)$. Ocho pacientes fueron retirados del estudio, 5 de los cuales por progresión de la enfermedad.

- En el estudio con triptorelina se incluyeron 120 pacientes con cáncer de próstata avanzado o con recidiva bioquímica después de tratamiento local. El $51,6 \%$ fueron $\mathrm{T} 3$, el $17,5 \%$ T4 y el $28,3 \%$ con recidiva bioquímica, solo el $5 \%$ tenían metástasis confirmadas. La mediana de PSA al inicio del estudio fue

\section{TABLA I. CARACTERISTICAS GENERALES DE LOS 3 ENSAYOS CLIINICOS.}

\begin{tabular}{|c|c|c|c|c|}
\hline & Tipo de estudio & Objetivo & Tamaño muestral & Criterios de inclusión \\
\hline LEUPRORELINA SC & $\begin{array}{l}\text { E.C. fase III, } \\
\text { multicéntrico, abierto }\end{array}$ & $\begin{array}{l}\text { Seguridad y } \\
\text { Eficacia }\end{array}$ & 111 pacientes & $\begin{array}{l}\text { - Diagnostico histológico } \\
\text { de cáncer } \\
\text { - Estadio mayor a T1 } \\
\text { - Estado general OMS 0-2 } \\
\text { - E. V. > } 1 \text { año }\end{array}$ \\
\hline TRIPTORELINA & $\begin{array}{l}\text { E.C. Fase III, } \\
\text { multicéntrico, abierto }\end{array}$ & $\begin{array}{l}\text { Seguridad, Eficacia } \\
\text { y Farmacodinámica }\end{array}$ & 120 pacientes & $\begin{array}{l}\text { - Diagnostico histológico } \\
\text { de cáncer } \\
\text { - Estadio localmente } \\
\text { avanzado o metastático. } \\
\text { - E.V. > } 18 \text { meses }\end{array}$ \\
\hline LEUPROLERINA IM & \begin{tabular}{|l} 
E.C. fase III, \\
multicéntrico, \\
aleatorizado a: \\
$-11,25 \mathrm{mg} / 3$ meses \\
$-22,5 \mathrm{mg} / 6$ meses \\
$-30 \mathrm{mg} / 6$ meses
\end{tabular} & $\begin{array}{l}\text { Seguridad y } \\
\text { Tolerabilidad }\end{array}$ & $\begin{array}{l}296 \text { pacientes } \\
-11,25 \mathrm{mg}: 58 \\
-22,5 \mathrm{mg}: 118 \\
-30 \mathrm{mg}: 120\end{array}$ & $\begin{array}{l}\text { - Diagnostico histológico } \\
\text { de cáncer } \\
\text { - Cualquier grado y estadio } \\
\text { Estado general OMS 0-3 } \\
\text { - Subsidiario de castración } \\
\text { endocrina } \\
\text { - E.V. > } 1 \text { año }\end{array}$ \\
\hline
\end{tabular}

E.C. - Ensayo Clínico; E.V. - Esperanza de Vida. 
de $19,1 \mathrm{ng} / \mathrm{ml}(0,1-1.630)$. Cinco pacientes fueron retirados del estudio, en 2 casos se produjo exitus por progresión de la enfermedad.

- En el estudio con leuprolerina IM se aleatorizaron 120 pacientes a la dosis semestral de $30 \mathrm{mg}, 58$ a la dosis trimestral de $11,25 \mathrm{mg}$ y 118 a la dosis semestral de $22,5 \mathrm{mg}$, estos últimos pacientes no fueron incluidos en los resultados finales del artículo del estudio (10). Los pacientes incluidos presentaban cáncer de próstata confirmado histológicamente de cualquier grado y estadio que "exigiera castración endocrina", una esperanza de vida superior a un año y un estado general OMS 0-3. El $21 \%$ habían recibido tratamiento previo con análogos de la LHRH. No se aportan datos sobre la estadificación tumoral. La mediana de PSA al inicio del estudio fue de 1,1 ng- $\mathrm{ml}^{-1}$. Se retiraron 7 pacientes, 4 por exitus y 3 por acontecimientos adversos graves.

\section{Análisis de la eficacia (Tabla II)}

- Leuprolerina SC

- El $97 \%$ de los pacientes alcanzaron niveles de castración (testosterona $<50 \mathrm{ng} / \mathrm{ml}$ ) a los 28 días de comenzar el tratamiento. Al final del estudio, el $99 \%$ de los pacientes tenían niveles de castración. En dos pacientes no se observó una supresión de la testosterona después de la administración del fármaco y antes de su retirada del estudio. No se detalla si existieron "escapes" de testosterona durante el estudio.

- Al final del estudio el PSA fue superior a $4 \mathrm{ng} / \mathrm{ml}$ en el 3,9\% de los pacientes, no existiendo cambios en los cuestionarios de dolor óseo y síntomas miccionales y no existió ninguna exacerbación clínica.

\section{- Triptorelina}

- El 97,5\% de los pacientes tenían niveles de castración (testosterona $<50 \mathrm{ng} / \mathrm{ml}$ ) a los 29 días de comenzado el estudio y el $93 \%$ lo mantuvo duran- te el estudio. Al final del estudio el 98,3\% mantenía una concentración de testosterona sérica en niveles de castración. Ocho $(6,7 \%)$ pacientes tuvieron "escapes" de testosterona durante el estudio (meses 2-12), en cinco de ellos el "escape" fue aislado sin repercusión en la concentración sérica del PSA.

- En $3(2,5 \%)$ pacientes se observó un aumento de la concentración de PSA con fracaso clínico.

- Leuprolerina IM

- El 96\% (1.257/1.310) de las concentraciones de testosterona realizadas durante el estudio fueron $\leq 50 \mathrm{ng} / \mathrm{dl}$. No se especifica el número o porcentaje de pacientes que presentaron "escapes" de testosterona.

- Al final del estudio se observó según los criterios de respuesta de la EORTC, remisión parcial en el $46,6 \%$, estabilización objetiva en el $46,6 \%$ y progresión objetiva n el 9,2\%. La reducción del PSA fue de $89 \%$ al final del estudio.

\section{Análisis de la seguridad (Tabla III)}

- Leuprolerina SC

- Los acontecimientos adversos más frecuentes fueron: sofocos $(33,3 \%$ leves y $24,3 \%$ moderados), reacción en el sitio de la inyección $114,4 \%$ leves y $0,9 \%$ moderado), cansancio $(7,2 \%$ leve y $4,5 \%$ moderado), atrofia testicular $(5,4 \%$ leve) y ginecomastia (3,6\% leve).

- Triptorelina

- Los acontecimientos adversos más frecuentes fueron: sofocos $(71,7 \%)$, disfunción eréctil $(10 \%)$, atrofia testicular $(7,5 \%)$ y reacción en el sitio de la inyección $(6,7 \%)$.

En el estudio con triptorelina se observó una mayor tolerabilidad local que en los estudios con leuprorelina (Figura 1).

TABLA II. SUPRESIÓN DE LOS NIVELES DE TESTOSTERONA PLASMÁTICA /<50 NG/MLI EN LOS 3 ESTUDIOS.

\begin{tabular}{|l|c|c|c|}
\hline & $1^{\mathrm{a}}$ mes & $1-12$ meses & Final estudio \\
\hline LEUPRORELINA SC & $97 \%$ & - & $99 \%$ \\
\hline TRIPTORELINA & $97,5 \%$ & $93 \%$ & $98,3 \%$ \\
\hline LEUPROLERINA IM & - & $96 \%$ & $90 \%$ \\
\hline
\end{tabular}


TABLA III. ACONTECIMIENTOS ADVERSOS EN LOS 3 ESTUDIOS ANALIZADOS.

\begin{tabular}{|l|c|c|c|c|c|c|c|}
\hline & Sofocos & $\begin{array}{c}\text { Reacción } \\
\text { Local }\end{array}$ & Cansancio & $\begin{array}{c}\text { Atrofia } \\
\text { testicular }\end{array}$ & Ginecomastia & $\begin{array}{c}\text { Disfunción } \\
\text { eréctil }\end{array}$ & $\begin{array}{c}\text { Aumento } \\
\text { sudoración }\end{array}$ \\
\hline LEUPRORELINA SC & $57,6 \%$ & $15,3 \%$ & $11,7 \%$ & $5,4 \%$ & $3,6 \%$ & - & - \\
\hline TRIPTORELINA & $71,7 \%$ & $6,7 \%$ & - & $7,5 \%$ & - & $10 \%$ & - \\
\hline LEUPROLERINA IM & $34,2 \%$ & $11,8 \%$ & $1,7 \%$ & - & - & - & $5,8 \%$ \\
\hline
\end{tabular}

- Leuprolerina IM

- El 15,8\% de los pacientes presentaron acontecimientos adversos graves, siendo retirado del estudio el $2,5 \%$ de los mismos.

- Los acontecimientos adversos más frecuentes fueron: sofocos $(34,2 \%)$, reacción en el sitio de la inyección $(11,8 \%)$, con presencia de induración en el $5,8 \%$ de los casos, aumento de la sudoración $(5,8 \%)$ y cansancio $(1,7 \%)$.

\section{DISCUSIÓN}

Los tres ensayos clínicos comparados son los "pivotal trials" que se presentaron a las autoridades reguladoras para su aprobación. La eficacia se mide según el porcentaje de pacientes que alcanzan el $1^{\text {er }}$ mes, durante el estudio y al final del mismo los valores de castración plasmática de testosterona que solicitan las autoridades reguladoras (<50 ng/dl.).

Una testosterona plasmática $<50 \mathrm{ng} / \mathrm{dl}$. se considera desde hace más de 40 años como el nivel de referencia estándar de castración. Recientemente algunos autores recomiendan que el nivel de testosterona plasmática se debería de aproximar lo más posible al nivel de castración quirúrgica $(20 \mathrm{ng} / \mathrm{ml})$ $(12,13)$.

Aunque existen algunas diferencias entre los 3 estudios en la forma de presentar los valores de testosterona plasmática (Tabla II), se puede considerar que no existen diferencias entre ellos ya que se alcanza un porcentaje de supresión de la testosterona $(<50 \mathrm{ng} / \mathrm{dl})$ entre el $93 \%$ y el $99 \%$. Independientemente de la formulación mensual o trimestral los análogos de la LHRH no consiguen en todos los pacientes una supresión de la testosterona. Entre el $2 \%$ y el $12,5 \%$ de los pacientes no conseguirán unos niveles de testosterona inferiores a $50 \mathrm{ng} / \mathrm{dl}$, en cambio entre el $13 \%$ y el $46,4 \%$ de los pacientes no alcanzaran niveles de testosterona inferiores a $20 \mathrm{ng} / \mathrm{dl}$ (14-18).

Los "escapes" de testosterona se definen como las elevaciones persistentes de testosterona por encima de $50 \mathrm{ng} / \mathrm{dl}$ a pesar de la administración continua de análogos de la LHRH. Para algunos autores estos "escapes" tienen un significado clínico y deberían de tener implicaciones terapéuticas $(16,19)$. La incidencia de "escapes" a largo plazo en los pacientes en tratamiento con análogos de la $\mathrm{LHRH}$ oscilan entre el $4 \%$ y el $12,5 \%(18,20,21)$. Recientemente Morote et al. (22) en un estudio retrospectivo observó la relación existente entre los "escapes" de testosterona y la supervivencia libre de progresión a andrógeno-independiente, cuanto mayor es el "escape" menor es la supervivencia libre de progresión.

Reacciones adversas locales (\%)

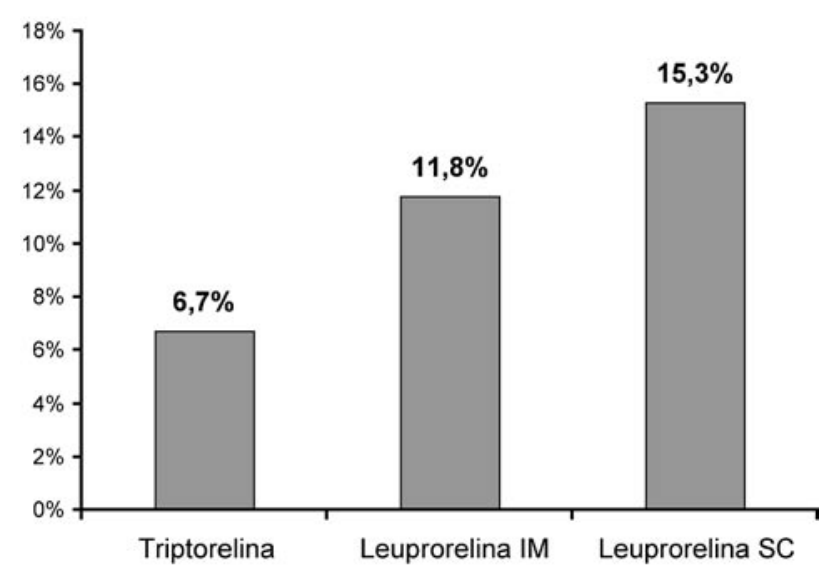

FiGURA 1. Análisis comparativo de la tolerabilidad local de los análogos semestrales de la LHRH. Porcentaje de reacciones adversas en el lugar de inyección. 
Solo el estudio con triptorelina describe detalladamente el porcentaje de "escapes" de testosterona y su repercusión en el PSA, el estudio con leuprorelina SC no hace ninguna referencia a los escapes y el estudio con leuprolerina IM enmascara el número de pacientes con "escapes" al analizar en bloque el número de determinaciones de testosterona durante el estudio y comunicar que un $4 \%$ de las misma fueron mayores a $50 \mathrm{ng} / \mathrm{dl}$.

Debido a la heterogeneidad de los pacientes incluidos en los 3 ensayos y a la variabilidad en la forma de presentar los resultados sobre los niveles de PSA al final del estudio, no es posible saber si existen diferencias en este sentido.

Los efectos adversos del tratamiento con análogos de la LHRH son debidos al hipogonadismo producido. A corto y medio plazo se observan sofocos, anemia, cansancio, disminución de la libido, disminución de la densidad ósea, aumento de la cantidad de grasa y disminución de la masa muscular. A largo plazo se ha descrito aumento del riesgo de diabetes y de enfermedades cardiovasculares (23).

En los 3 estudios se comunicaron un elevado porcentaje de acontecimientos adversos, siendo la mayoría de ellos leves. En el estudio de triptorelina y en el de leuprorelina SC no hubo retiradas del estudio debido a acontecimientos adversos graves (AAG), mientras que el $2,5 \%$ de los pacientes del estudio de leuprolerina IM fue retirado del estudio debido a AAG.

A pesar de que en los 3 estudios existe una importante variabilidad del análisis y la forma de expresar los datos, no parece que existan grandes diferencias en cuanto a eficacia y seguridad. La realización de un estudio aleatorizado entre estos 3 principios activos sería la única forma de detectar, si existieran, diferencias entre ellos.

\section{NOTA}

Este estudio ha sido financiado por Ipsen Pharma S.A.

\section{BIBLIOGRAFÍA y LECTURAS} RECOMENDADAS (*lectura de interés $y^{* *}$ lectura fundamental)

1. Jemal A, Siegel R, Ward E, Hao Y, Xu J, Thun MJ. Cancer Statistics, 209. Ca Cancer J Clin. 2009; 59:225-49.

**2. Herranz Amo F, Arias Fúnez F, Arrizabalaga Moreno M, Calahorra Fernández FJ, Carballido Rodríguez J, Diz Rodríguez R, et al. El cáncer de próstata en la Comunidad de Madrid en el año 2000. II - Presentación y diagnóstico. Actas Urol Esp, 2003; 335-44.

3. Heindenreich A. Bolla A, Joniau S, van der Kwast $\mathrm{TH}$, Matveev V, Mottet MN, et al. Guidelines on prostate cancer. 2009. http://www.uroweb. org/fileadmin/tx_eauguidelines/2009/Full/Prostate_Cancer.pdf.

4. NCCN Clinical Practice Guidelines in Oncology. Prostate Cancer. v.2.2009. www.nccn.org.

5. Barry MJ, Delorenzo MA, Walker-Corkery ES, Lucas FL, Wennberg DC. The rising prevalence of androgen deprivation among older American men since the advent of prostate-specific antigen testing: a population-based cohort study. BJU Int, 2006; 98:973-8.

6. Huggins C, Hodges CV. Studies on prostate cancer. Effect of castration, estrogen and androgen injection on serum phosphatases in metastatic carcinoma og the prostate. Cancer Res. 1941. 1:293-7.

7. Lahlou N. Pharmacocinétique et pharmacodynamique de la triptoreline. Ann Urol. 2005. 39:S78S84.

8. McLeod DG. Hormonal therapy: Historical perspective to future directions. Urology. 2003. 61(Suppl 2A): 3-7.

**9. Crawford ED, Sartor O, Chu F, Perez R, Karlin G, Garrett JS. A 12-month clinical study of LA-2585 $(45.0 \mathrm{mg})$ : A new 6-months subcutaneous delivery system for leuprolide acetate for the treatment of prostate cancer. J Urol, 2006; 175:533-6.

**10. Tunn UW, Wiedey K. Safety and clinical efficacy of a new 6-month depot formulation of leuprolerin acetate in patients with prostate cancer in Europe. Prostate Cancer Prostatic Dis, 2009; 12:83-7.

**11. Lundström EA, Rencken RK, van Wyk JH, Coetzee LJE, Bahlmann JCM, Reif S, et al. Triptorelin 6-months formulation in the management of patients with locally advanced and metastatic prostate cancer. An open-label, non-comparative, multicentre, phase III study Clin Drug Investig, 2009; 29:757-65.

*12. Oefelein MG, Feng A, Scolieri MJ, Ricchiuti D, Resnick MI. Reassessment of the definition of castrate levels of testosterone: implications for clinical decision making. Urol, 2000; 56:1021-4. 
*13. Morote J, Orsola A, Planas J, Trilla E, Raventós CX, Cecchini L, Catalán R. Redefining clinically significant castration levels in patients with prostate cancer receiving continuous androgen deprivation therapy. J Urol, 2007; 178(4Pt1):1290-5.

*14. Morote J, Esquena S, Abascal JM, Trilla E, Cecchini L, Raventós CX, Catalán R, Reventós J. Failure to maintain a suppressed level of serum testosterone during long-acting depot luteinizing hormone-releasing hormone agonist therapy in patients with advanced prostate cancer. Urol Int, 2006; 77:135-8.

15. Sarosdy MF, Schellhammer PF, Soloway MS, Vogelzang NJ, Crawford ED, Presti J, Chodak GW, Mitchell P, Porter L. Endocrine effects, efficacy and tolerability of a 10.8-mg depot formulation of goserelin acetate administered every 13 weeks to patients with advanced prostate cancer. BJU Int, 1999; 83:801-6.

*16. Oefelein MG, Cornum R. Failure to achieve castrate levels of testosterone during luteinizing hormone releasing hormone agonist therapy: the case for monitoring serum testosterone and a treatment decision algorithm. J Urol, 2000; 164(3 Pt 1):7269.

17. Wechsel HW, Zerbib M, Pagano F, Coptcoat MJ. Randomized open labelled comparative study of the efficacy, safety and tolerability of leuprorelin acetate $1 \mathrm{M}$ and $3 \mathrm{M}$ depot in patients with advan- ced prostatic cancer. Eur Urol, 1996; 30 (Suppl 1):7-14.

18. Jocham D. Leuprorelin three-month depot in the treatment of advanced and metastatic prostate cancer: long-term follow-up results. Urol Int, 1998; 60 (Suppl 2):18-24.

*19. Zlotta A, Debruyne FMJ. Expert opinion on optimal testosterone control in prostate cancer. Eur Urol, 2005; (Supple. 4): 37-41.

20. Khan MS, O’Brien A. An evaluation of pharmacokinetics and pharmacodynamics of leuprorelin acetate 3M-depot in patients with advanced and metastatic carcinoma of the prostate. Urol Int, 1998; 60:33-40.

21. Fontana D, Mari M, Martinelli A, Boccafoschi C, Magno C, Turriziani M, et al. 3-month formulation of goserelin acetate ('Zoladex' 10.8-mg depot) in advanced prostate cancer: results from an Italian, open, multicenter trial. Urol Int, 2003; 70:316-20.

*22. Morote J, Orsola A, Planas J, Trilla E, Raventós CX, Cecchini,L, et al. Redefining clinically significant castration levels in patients with prostate cancer receiving continuos androgen deprivation therapy. J Urol, 2007; 178:1290-5.(*)

23. Michaelson MD, Cotter SE, Gargollo PC, Zietman AL, Dahl DM, Smith MR. Management of complications of prostate cancer treatment. CA Cancer J Clin, 2008; 58:196-213. 